\title{
Effect of long term inhaled corticosteroid therapy on adrenal suppression, growth and bone health in children with asthma
}

Anuradha KWDA ${ }^{1,2^{*}}$ (D) Prematilake $\mathrm{GLDC}^{3}$, Batuwita BAUI ${ }^{4}$, Kannangoda KASR ${ }^{5}$, Hewagamage US ${ }^{6}$, Wijeratne $\mathrm{S}^{7}$, Lankatilake Kantha ${ }^{8}$ and de Silva $\mathrm{KSH}^{9}$

\begin{abstract}
Background: Inhaled corticosteroids (ICS) are the most effective treatment for children with persistent asthma. However adverse effects of ICS on Hypothalamo Pituitary Adrenal (HPA) axis, growth and bone metabolism are a concern. Hence the primary objective of this study was to describe the effects of long term inhaled corticosteroid therapy (ICS) on adrenal function, growth and bone health in children with asthma in comparison to an age and sex matched group of children with asthma who were not on long term ICS. Describing the association between the dose of ICS and duration of therapy on the above parameters were secondary objectives.

Method: Seventy children with asthma on ICS and 70 controls were studied. Diagnosis of asthma in selected patients was reviewed according to the criteria laid down by GINA 2018 guidelines. The estimated adult heights were interpreted relative to their Mid Parental Height (MPH) range. Serum calcium, alkaline phosphatase and vitamin D levels were analyzed in both groups and cortisol value at 30 min following a low dose short synacthen test was obtained from the study group. The average daily dose of ICS (Beclamethasone) was categorized as low, medium and high (100-200, 200-400, > $400 \mu \mathrm{g} /$ day) respectively according to published literature.

Results: Heights of all children were within the MPH range. There was no statistically significant difference in the bone profiles and vitamin D levels between the two groups (Ca: $p=0.554$, vitamin $D: p=0.187$ ) but vitamin $D$ levels were insufficient $(<50 \mathrm{nmol} / \mathrm{l})$ in $34 \%$ of cases and $41 \%$ of controls. Suppressed cortisol levels were seen in $24 \%$. Doses of ICS were low, medium and high in 56, 32 and 12\% of children respectively. The association between adrenal suppression with longer duration of therapy $(p<0.01)$ and with increasing dose of ICS $(p<0.001)$ were statistically significant.
\end{abstract}

Conclusion: ICS had no impact on the growth and bone profiles but its dose and duration were significantly associated with adrenal suppression.

Keywords: Asthma, Inhaled corticosteroids, Growth, Adrenal suppression, Bone metabolism, Vitamin D

\section{Background}

Asthma is a commonly encountered chronic inflammatory disease in children where there is an airway hyper responsiveness leading to obstruction and limitation of the airflow. It is clinically defined by the presence of recurrent respiratory symptoms such as cough, wheeze,

\footnotetext{
* Correspondence: anujaya2008@gmail.com

${ }^{1}$ Lecturer Faculty of Medicine, University of Colombo, Colombo, Sri Lanka

${ }^{2}$ Acting paediatric pulmonologist, University paediatric unit, Lady Ridgeway Hospital for children, Colombo, Sri Lanka

Full list of author information is available at the end of the article
}

shortness of breath and chest tightness that vary over time and in intensity with variable expiratory airflow limitation [1].

Inhaled corticosteroids (ICS) are considered to be the most effective treatment for management of persistent asthma as they prevent exacerbations, improve lung function and the quality of life and reduce hospitalizations and asthma related mortality [2]. Although the side effects of ICSs are less frequent and severe than that of oral corticosteroids, safety concerns with these agents still remain especially in relation to high doses [3].

(c) The Author(s). 2019 Open Access This article is distributed under the terms of the Creative Commons Attribution 4.0 International License (http://creativecommons.org/licenses/by/4.0/), which permits unrestricted use, distribution, and 
Effects of ICS on growth of children, bone health and adrenal function are the key areas of concern in studies found in the literature.

Possible suppression of the Hypothalamo-Pituitary Adrenal (HPA) axis leading to adrenal suppression (AS) is an under recognized, life threatening adverse effect $[4,5]$. It is a proven complication of most forms of glucocorticoid therapy that persist even after one year of cessation of therapy [6]. Therefore screening of patients for AS is important. This is dependent on many factors such as the type of corticosteroid, duration of therapy and the dose which is proportional to the degree of suppression.

Corticosteroid induced enhancement of osteoclastic activity and suppression of osteoblasts leading to bone resorption is presumed to affect growth and bone metabolism $[7,8]$.

\section{Objectives}

The primary objective was to describe the effect of long term inhaled corticosteroid therapy (ICT) on adrenal suppression, growth and bone health in children with Asthma.

Secondary objectives were to

- Describe the association between the dose of inhaled corticosteroids and duration of therapy on adrenal functions, growth and bone health in children with asthma

- Compare the growth and effect on the biochemical parameters associated with bone health between children with asthma who are on ICS and age and sex matched group of children with asthma who were not on long term ICT.

- Estimate and compare vitamin D levels in both groups of children and assess the impact of ICS on Vitamin D levels.

\section{Method}

Children aged 3 to 9 years with asthma on long term inhaled corticosteroids for at least 6 months were studied with an age and sex matched comparison group of children with asthma who were not on ICS. A cross sectional descriptive study was conducted at the Asthma Clinic of the University Paediatric Unit of Lady Ridgeway Hospital (LRH), Colombo, Sri Lanka from September 2016 to April 2018.Children older than 9 years were excluded to avoid the pre-adolescent physiological acceleration of growth seen at this age. Because of the possibility of patients with bronchiolitis being included and as nutrition was the main determinant of growth, children under 3 years were not included in the study. Patients who had been on medication for asthma during the week preceding the study were excluded due to the possibility of being given oral steroids which may have interfered with the evaluation of the adrenal axis. Those having long standing illnesses with possible impact on the parameters being evaluated were also excluded from the study. The objectives of this study being to assess the possible adverse effects of ICS related to its dose and duration, the severity and the control of asthma in selected children was not considered in their enrollment.

A convenient sample of 70 children each in the two groups, were studied. Ethical clearance was obtained from the Ethics Review Committees of the Faculty of Medicine, University of Colombo and Lady Ridgeway Hospital, Colombo. Written informed consent was obtained prior to the study, from the mother/guardian and assent when appropriate from the patients.

Socio-demographic and treatment details were recorded using an interviewer administered questionnaire. Heights of the participants were measured using a stadiometer to the nearest $0.1 \mathrm{~cm}$ according to the stipulated recommendations. The heights of both parents were also recorded. The mid parental height or target height (TH) was calculated for each patient using the formula given below [9].

Boy $=$ mother's height + father's height $+13.0 \mathrm{~cm} \div 2$.

Girl $=$ mother's height + father's height $-13.0 \mathrm{~cm} \div 2$.

Mid parental height range $=\mathrm{TH} \pm 8.5 \mathrm{~cm}$.

The mid parental height range was plotted on the percentile chart at the point of 18 years in each patient. The current height of each child were projected up to 18 years (which is assumed to be the adult height [9] and recorded in relation to the MPH range.

Two milliliters of venous blood was drawn after $6 \mathrm{~h}$ of fasting at 8 am from both the study sample and control group, for Serum Calcium, Phosphate, Alkaline Phosphatase (ALP) and Vitamin D. The assay included serum cortisol in the study sample.

Evidence of adrenal suppression was assessed in the study sample by the low dose short Synacthen test. After taking blood for the biochemical analyses, 1 microgram of Synacthen (soluble ACTH) was administered intravenously and a blood sample was taken 30 min later for serum cortisol to assess the function of HPA axis. Peak Cortisol level $>500 \mathrm{nmol} / \mathrm{L}$ or $200 \mathrm{nmol} / \mathrm{L}$ increment from the basal cortisol level was considered as the normal response [10]. Relationship of adrenal suppression to the dose of ICS was analyzed with the age specific categorization of the cumulative dose of ICS received by children in to high, medium and low dose according to GINA guidelines [1]. Evaluation software, SPSS version 20 was used for data analysis.

\section{Results}

Adrenal suppression

A peak cortisol level of $<500 \mathrm{nmol} / \mathrm{L} 30 \mathrm{~min}$ after the low dose adreno-corticotropin test, indicating adrenal 
suppression and therefore an effect on the HPA,was seen in $17(24.3 \%)$ of the study population. ICS had been used for more than 24 months in 13 (76.5\%) of the 17 patients in whom adrenal suppression was documented (Table 1).

An increase in adrenal suppression was noted with the increasing duration of therapy. This association between duration of corticosteroid therapy and adrenal suppression was found to be statistically significant. $\left(X^{2}=12.291\right.$; $\mathrm{df}=3 ; p<0.01)$.

Ten $(58.8 \%)$ of the 17 patients with adrenal suppression had received high doses of ICS (Table 2). The association between the dose of inhaled corticosteroid therapy and adrenal suppression was statistically significant $\left(\mathrm{X}^{2}=29.80 ; \mathrm{df}=2 ; \mathrm{p}<0.01\right)$.

\section{Height}

Effects of long term corticosteroids on the stature of children was assessed by comparing the heights of the children in the two groups in relation to their mid parental height $(\mathrm{MPH})$ range.

No child's height was below the MPH in either of the groups (Table 3). The association between use of long term inhaled corticosteroids and growth in terms of height was not statistically significant $(\mathrm{X} 2=0.785 ; \mathrm{df}=1$; $p=0.376)$.

\section{Bone health}

Calcium and ALP values of the two groups of children did not show a normal distribution thus the Independent samples Mann-Whitney U Test was applied to test for the significance of the results.

The difference between the median calcium values of the study and control groups was not statistically significant $(p=0.88)$. Median ALP values of the study and control groups were $225 \mathrm{U} / \mathrm{L}$ and $198.5 \mathrm{U} / \mathrm{L}$ respectively, and this difference was statistically significant $\{(p<0.01)$, (Table 4)\}.

\section{ICS and vitamin D}

According to Independent samples Mann - Whitney U Test (Table 5), no significant association was found between long term ICT and vitamin D levels $(p=0.886)$.

Table 1 Relationship between ICS duration and adrenal suppression $(n=70)$

\begin{tabular}{llll}
\hline $\begin{array}{l}\text { Duration of ICS } \\
\text { therapy (months) }\end{array}$ & \multicolumn{2}{l}{ Adrenal suppression } & Total \\
\cline { 2 - 3 } & Not suppressed & Suppressed & \\
\hline$<12$ & 9 & 0 & 9 \\
$12-18$ & 4 & 1 & 5 \\
$18-24$ & 0 & 3 & 3 \\
$>24$ & 40 & 13 & 53 \\
Total & $53(75.7 \%)$ & $17(24.3 \%)$ & $70(100 \%)$ \\
\hline
\end{tabular}

Table 2 Relationship between ICS dose and adrenal suppression $(n=70)$

\begin{tabular}{llll}
\hline Dose of ICS $(\mu \mathrm{g} /$ day $)$ & \multicolumn{2}{l}{ Adrenal Suppression } & Total \\
\cline { 2 - 3 } & Not suppressed & Suppressed & \\
\hline High $(>400 \mu \mathrm{g} /$ day $)$ & 2 & 10 & 12 \\
Medium $(200-400 \mu \mathrm{g} /$ day $)$ & 23 & 6 & 29 \\
Low $(100-200 \mu \mathrm{g} /$ day $)$ & 28 & 1 & 29 \\
Total & 53 & 17 & 70 \\
\hline
\end{tabular}

Interestingly $34 \%$ of the control group and $41 \%$ of the study group showed insufficient vitamin D values $(\leq 50$ $\mathrm{nmol} / \mathrm{L}$ ) and $11 \%$ of the control group and $4 \%$ of the study group showed vitamin D deficiency $(\leq 30 \mathrm{nmol} / \mathrm{L})$.

\section{Discussion}

Identifying the potential adverse effects of inhaled corticosteroids on growth, bone metabolism and the HPA axis had been the focus in many reports in international literature where these aspects had been studied separately. Similar studies have not been reported previously from Sri Lanka. The results of this study demonstrated that long term ICS therapy had a significant effect on adrenal suppression but not on bone health and growth.

\section{ICS and adrenal suppression}

The most serious potential complication of ICS is adrenal insufficiency hence prevention and screening of patients for adrenal suppression (AS) is vital [6].

Endogenous cortisol production is suppressed by exogenous glucocorticoids. When exogenous glucocorticoids significantly alter the pattern of normal diurnal variation in cortisol levels adverse consequences are expected [12]. Acute adrenal insufficiency was seen less commonly compared to adrenal suppression shown by laboratory tests [13].

In our study $24.3 \%$ of patients on ICS showed a peak cortisol level $<500 \mathrm{nmol} / \mathrm{L}$ at $30 \mathrm{~min}$ after the low dose ACTH test indicating AS. This was significantly associated with increasing doses and duration of ICS therapy (Tables 1 and 2). High cumulative dose of ICS $(>400 \mu \mathrm{g} /$ day) had been received by $58.8 \%$ of children who showed adrenal suppression and $76.5 \%$ had been on treatment for more than 2 years .

In a survey conducted in UK, 33 patients (28 children, 5 adults) were identified to have adrenal crisis. Out of children 23 had presented with symptomatic hypoglycemia

Table 3 Heights of children relative to MPH range $(n=140)$

\begin{tabular}{llll}
\hline Height & Control group & Study Group & Total \\
\hline Within MPH range & $43(61.4 \%)$ & $48(68.6 \%)$ & $91(65.0 \%)$ \\
$>$ MPH range & $27(38.6 \%)$ & $22(31.4 \%)$ & $49(35.0 \%)$ \\
Total & $70(100.0 \%)$ & $70(100.0 \%)$ & $140(100.0 \%)$ \\
\hline
\end{tabular}


Table 4 Comparison of Calcium and ALP values in the two groups $(n=140)$

\begin{tabular}{llllll}
\hline & \multicolumn{2}{l}{ Control group (70) } & & \multicolumn{2}{l}{ Study group (70) } \\
\cline { 2 - 3 } \cline { 5 - 6 } & Median value & IQR (Inter Quartile Range) & & Median value & IQR (Inter Quartile Range) \\
\hline Calcium $(\mathrm{mmol} / \mathrm{L})$ & 2.32 & $2.25-2.37$ & 2.35 & $2.26-2.44$ \\
ALP $(\mathrm{U} / \mathrm{L})$ & 198.5 & $147.50-233.25$ & 225 & $208.25-283.00$ \\
\hline
\end{tabular}

and all of them were treated with 500-2000 $\mu$ g/day ICS. Majority $(76 \%)$ of the patients were treated for at least a year [14].

A Finnish study had randomly divided 75 children on ICS into three treatment groups according to the different types of corticosteroids used and performed the low dose ACTH test which had demonstrated AS in $23 \%$ of the children using moderate doses of inhaled steroids [15].

Zollner et al had reported AS in 35 to $65 \%$ of children on ICS $\geq 500 \mu \mathrm{g} /$ day for up to 16 weeks after starting therapy but no significant AS had been detected with low doses [16].

\section{ICS and growth}

In this study the doses of ICS used were low (100-200 $\mu \mathrm{g} /$ day), medium (200-400 $\mu$ g/day) and high $(>400 \mu \mathrm{g} /$ day) in $41.4,41.4$ and $17.1 \%$ of children respectively and there was no significant difference in height in relation to the MPH range between the study and control groups.

A study done on the adverse effects on growth in patients given ICS for more than 1 year had shown a statistically significant effect on pre-pubertal children but not on pubertal school children [17]. A one-year prospective cohort study carried out in 2001on 124 asthmatic children aged 3 to 16 years on ICS for at least 12 months, the $\mathrm{z}$-scores for height for age, weight for age, body mass index and parental target height for current age concluded that there was no significant effect on the height or body weight of children/adolescents using ICS for more than 1 year at the doses recommended for prevention of asthma [18].

Although no formal dose-response studies have been conducted, data from a European study suggests that the effect of ICS on linear growth is dose dependent. A daily dose of $800 \mu \mathrm{g}$ was found to have a significantly greater growth suppressive effect with a height velocity of 3.6 $\mathrm{cm} /$ year compared to a velocity of $4.6 \mathrm{~cm} /$ year at a dose of $400 \mu \mathrm{g} /$ day [18].
Efthimiou J et al (1998) in their systemic review concluded that the doses of inhaled corticosteroids up to $400 \mu \mathrm{g} /$ day in children have no significant effect on bones and growth in the large majority of patients with asthma [19]. In a study on prepubescent children with mild to moderate persistent asthma, a small but statistically significant group difference in growth velocity was observed between low doses of ICS and low to medium doses of a Beclamethasone equivalent, favoring the use of low-dose ICS [20]. Similarly, when ICS (Beclamethasone diproprionate) was used on a daily dose of 300 to $800 \mu \mathrm{g}$, the mean height velocity decreased when the dose was increased and when decreased or stopped, an increase in the mean height velocity was seen without a reduction in the final height. Thus, a careful assessment of height velocity in all children receiving ICS is recommended [21]. MPH range also provides a useful guide to predict the height percentile for children of parents of average stature [22].

The type of drug or the molecule and the aerosol generating device may alter the magnitude of the adverse effects of ICS on children with persistent asthma. Axelsson I et al (2019) in their review has compared different ICS drugs or inhalation devices with regard to their effects on growth velocity and suggest that they may impact the effect size on growth. Least impact on growth was observed with Fluticasone as the ICS and Easy haler as the delivery device.. However the authors suggest further studies before any recommendation is made on selection of the drug or the delivery devices [23] .In our study we made sure that all the children use the same delivery device at the enrollment and 67 children out of 70 who were on ICS were on Beclamethasone which is the available and commonly prescribed molecule at our setup.

\section{Bone metabolism}

Increased osteoclastic activity and suppression of osteoblastic activity of corticosteroids lead to accelerated bone resorption. Corticosteroids also enhance the inactivation

Table 5 Comparison of vitamin D levels in the two groups $(n=140)$

\begin{tabular}{|c|c|c|c|c|}
\hline & \multicolumn{2}{|c|}{ Control group (70) } & \multicolumn{2}{|c|}{ Study group (70) } \\
\hline & Median value & IQR (Inter Quartile Range) & Median value & IQR (Inter Quartile Range) \\
\hline Vitamin D value (nmol/L) & 50.5 & $44.32-67.00$ & 53.75 & $44.25-65.75$ \\
\hline \multicolumn{5}{|c|}{ Vitamin D status: Deficient $\leq 30 \mathrm{nmol} / \mathrm{L}$, Insufficient $=30-50 \mathrm{nmol} / \mathrm{L}$, Sufficient $\geq 50 \mathrm{nmol} / \mathrm{L}$ [11] } \\
\hline
\end{tabular}


of vitamin $\mathrm{D}$ by up-regulating 24-hydroxylase activity. It degrades the physiologically active form of vitamin $D_{3}, 1$, 25-dihydroxycholecalceferol by hydroxylation of the side chain thereby increasing the risk of bone resorption. These actions of corticosteroids are important considerations as vitamin $D$ plays a significant role in bone mineralization $[6,7,24]$. Systemic side effects such as dose related reduction in bone density demonstrated by biochemical markers occur with ICS but are less compared to oral steroids [2]. A significant difference in serum calcium and phosphate between the two groups could not be seen but ALP in the study group was higher and the difference was statistically significant $(p<0.01)$.

Bone Mineral Density (BMD) is a sensitive marker of the effects of corticosteroids on bone and can be used to predict the fracture risk [25]. Decreased BMD has been demonstrated with high doses of ICS but not with low to medium doses [26, 27]. A study on 48 prepubertal children aged 5 to 14 years who were on 400 to $2000 \mu \mathrm{g} /$ day of beclomethasone dipropionate Metered Dose Inhaler (MDI) has shown slow BMD acquisition in children who were on high doses and no effects with low to moderate doses [28]. Similarly, a case control study [29] and a study by the Childhood Asthma Management Programme (CAMP) group did not show a significant effect on bone density with low to medium doses of ICS [30].

Facilities to evaluate BMD were not available at LRH at the time of this study. Therefore serum calcium, phosphate and alkaline phosphatase (ALP) as biochemical markers of bone health were compared in the two groups.

\section{ICS and vitamin D}

The results of this study revealed no significant association between long term inhaled corticosteroid therapy and vitamin D level $(p=0.886)$ but vitamin D deficiency was seen in $13(18 \%)$ and $6(8 \%)$ of the control and study groups respectively.

There is growing evidence that vitamin D plays a role in the pathogenesis of allergic diseases and asthma but it is unclear whether supplementation during childhood may improve the outcome. There is evidence of reduction in active vitamin D levels with the use of corticosteroids $[6,7,22]$. Della Giustina A et al in their review had analyzed the possible links between vitamin D and its supplementation in allergic diseases and concluded that the current evidence to support vitamin D supplementation for the prevention or treatment of allergic diseases including asthma in children is insufficient [31].

\section{Limitations}

Being a descriptive cross-sectional study, this study was unable to demonstrate the effects on growth velocity which was a limitation. Serum Parathyroid Hormone levels were not assayed, which would have been useful to arrive at a more accurate interpretation of the biochemical markers, which is another limitation of this study.

\section{Conclusions and recommendations}

This study concludes that inhaled corticosteroids can cause adrenal suppression when administered in high doses ( $>400 \mu \mathrm{g} /$ day) and for longer durations. ( $>18$ to 24 months). Close follow up of the patients with regular assessment of the growth and asthma control would enable the dose of ICS to be gradually reduced to the lowest possible dose needed for optimum control of symptoms. A significant effect on the growth of the children who were on ICS was not demonstrated from the findings of this study.

The recommendations proposed from this study would be to pay meticulous attention based on the clinical response to treatment and to be vigilant about the possibility of adrenal suppression when asthma is treated in children.

\section{Abbreviations}

ACTH: Adreno Corticotropic Hormone; ALP: Alkaline Phosphatase:

AS: Adrenal Suppression; BMD: Bone Mineral Density; HPA axis: Hypothalamo Pituitary Adrenal axis; ICS: Inhaled Cortico Steroids; ICT: Inhaled Corticosteroid Therapy; LRH: Lady Ridgeway Hospital; MDI: Metered Dose Inhaler; MPH: Mid Parental Height; TH: Target Height

\section{Acknowledgements}

$>$ All the CO- authors of the study who contributed in protocol generation data collection, data entering, data analysis and reviewing the manuscript.

$>$ All the patients and their parents who voluntarily participated in the study.

$>$ Nursing staff and the other staff of the University paediatric unit of the Lady Ridgeway Hospital who assisted in blood drawing and sample handling.

$>$ Laboratory staff of the Lady Ridgeway Hospital for Children and Vindana Reproductive center.

\section{Authors' contributions}

KWDAA - Principal investigator, Conception of the study, Protocol Generation, Data Collection and Manuscript Writing. GLDCP- Co investigator,Provision of inputs for the endocrine aspects for the protocol, Performance of short synacthen test and analysis of results, Reading and approving the Manuscript. BAUI- Data collection, Data entering and analysis. Reading and approving the manuscript. KASR- Protocol generation, Data collection and data entering. Reading and approving the manuscript. USHProtocol generation, Data collection and data entering. Reading and approving the manuscript. WS- Reviewing the protocol, Performance of laboratory investigations done in the study. Reading and approving the manuscript. KL-Provision of inputs for the statistical aspects of the protocol, Statistical analysis of the results, Editing and approving the Manuscript. KSHDS - Overall supervisor, Conception of the study, reviewing and editing the Protocol, Supervision of the study, Data Analysis, Reviewing and final editing of the manuscript. All authors read and approved the final manuscript.

Funding

○ Self-funded

Availability of data and materials

- The datasets used for analysis during the current study are available from the corresponding author on request. All the relevant results are given in the manuscript. Can submit to BMC pediatrics if expected to sumbit. 


\section{Ethics approval and consent to participate}

$>$ Ethical approval was obtained from the following Ethical Review Committees Faculty of Medicine University of Colombo- Ref No.EC-17-067 Lady Ridgeway Hospital for children, Colombo

$>$ All the parents of the children and older children who participated in the study were given the information sheets with the details of the study in their language approved by the ethical review committee. Written informed consents for participation were obtained from the parent/ guardian of the children.

\section{Consent for publication}

- This manuscript does not contain personal data/ images/ videos of any individual person, therefore consent for publication is not applicable according to the publication guidelines of BMC paediatrics.

\section{Competing interests}

o No financial or non-financial competing interests related to this study.

\section{Author details}

${ }^{1}$ Lecturer Faculty of Medicine, University of Colombo, Colombo, Sri Lanka.

${ }^{2}$ Acting paediatric pulmonologist, University paediatric unit, Lady Ridgeway Hospital for children, Colombo, Sri Lanka. ${ }^{3}$ Acting paediatric endocrinologist, Lady Ridgeway Hospital for children, Colombo, Sri Lanka. ${ }^{4}$ Research Assistant, University paediatrc unit, Lady Ridgeway Hospital for Children, Colombo, Sri Lanka. ${ }^{5}$ Medical Officer, National Hospital of Sri Lanka, Colombo, Sri Lanka. ${ }^{6}$ Medical Officer, Lady Ridgeway Hospital for Children, Colombo, Sri Lanka. ${ }^{7}$ Laboratory Director, Vindana Reproductive Health Centre, Colombo, Sri Lanka. ${ }^{8}$ Associate Professor, Department of Community Medicine, Faculty of Medicine, University of Colombo, Colombo, Sri Lanka. ${ }^{9}$ Professor in paeditrics ,Faculty of Medicine, University of Colombo and Lady Ridgeway Hospital for Children, Colombo, Sri Lanka.

Received: 5 July 2019 Accepted: 9 October 2019

\section{Published online: 05 November 2019}

\section{References}

1. Global Initiative For Asthma. (2017). Global strategy for asthma management and prevention. [online] available at:https:/ginasthma.org/ wp-content/uploads/2018/04/wms-GINA-2018-report-tracked_v1.3. pdf[accessed 15 Feb 2019].

2. Enrico H, Lorena N, Girardi M, Matteo F, Francesca P, Luca M, Giorgio GP. Inhaled Corticosteroids Safety and Adverse Effects in Patients with Asthma. The Journal of Allergy and Clinical Immunology in Practice. 2008;6-3:776-81.

3. Nicola A, Kenneth RC, William CS, John PS, Steven K. Dose-related decrease in bone density among asthmatic patients treated with inhaled corticosteroids. J Allergy Clin Immunol. 2005:571-9.

4. Karine IEK. CSACI position statement: systemic effect of inhaled corticosteroids on adrenal suppression in the management of pediatric asthma. Allergy Asthma, and Clinical Immunology. 2015;11:1-9.

5. Ronald D. Systemic side effects of inhaled corticosteroids in patients with asthma. Respir Med. 2006;100:1307-17.

6. Alexandra A, Harold K, Sheldon S. Adrenal suppression :A practical guide to the screening and management of this under-recognized complication of inhaled corticosteroid therapy. Allergy Asthma and Clinical Immunology. 2011;7:13.

7. Kurahashi I, Matsunuma A, Kawane T, Abe M, Horiuchi N. Deaxamethasone enhances vitamin D-24-hydroxylase expression in osteoblastic (UMR-106) and renal $\left(L L C-P K_{1}\right)$ cells treated with 1a,25-dihyroxyvitamin $D_{3}$. Endocrine. 2002;17:109-18.

8. Dhawan $\mathrm{P}$, Christakos S. Novel regulation of 25-hydroxyvitamin $\mathrm{D}_{3}$ 24hydroxylase $24(\mathrm{OH})$ ase transcription by glucocorticoids: cooperative effects of the glucocorticoid receptor, $C / E B P \beta$, and the vitamin $D$ receptor in 24(OH) ase transcription. J Cell Biochem. 2010;1 10:1314-23.

9. Tanner JM, Goldstein H, Whitehouse RH. Standards for children's height at ages 2-9 years allowing for height of parents. Archives of Disease in Childhood. 1970;45:755-62.

10. Park J, Mohammed D, Joanne B. The diagnosis and treatment of adrenal insufficiency during childhood and adolescence. Arch Dis Child. 2016;101:860-5.
11. Craig F, Munns N, Bonny L. Global consensus recommendations on prevention and Management of Nutritional Rickets. Horm Res Paediatr. 2016;85:83-106.

12. Allen DB. Sense and sensitivity: assessing inhaled corticosteroid effects on the hypothalamic- pituitary-adrenal axis. Ann Allergy Asthma Immunology. 2002;89:537-9.

13. Santiago AH, Ratzan S. Acute adrenal crisis in an asthmatic child treated with inhaled fluticasone proprionate. Int J Pediatr Endocrinol. 2010;749239. https://doi.org/10.1155/2010/749239.

14. Drake AJ, Howells RJ, Shield JPH, Prendiville A, Ward PS, Crowne EC. Symptomatic adrenal insufficiency presenting with hypoglycaemia in asthmatic children with asthma receiving high dose inhaled fluticasone propionate. Br Med J. 2002;324(7345):1081-2.

15. Kannisto S, Korppi M, Remes K, Voutilainen R. Adrenal suppression, evaluated by a low dose adrenocorticotropin test, and growth in asthmatic children treated with inhaled steroids. J Clin Endocrinol Metab. 2000;85(2): 652-7.

16. Zollner EW, Lombard CJ, Galal U, Hough S, Irusen EM, Weinberg E. Hypothalamic-pituitary-adrenal axis suppression in asthmatic school children. Pediatrics. 2012;130:e1512.

17. Agertoft $L$, Pedersen $S$. Effect of long-term treatment with inhaled budesonide on adult height in children with asthma. New England Journel Medicine. 2000;343:1064-9.

18. Pedersen S. Do Inhaled Corticosteroids Inhibit Growth in Children? American Journal of Respiratory and Critical Care Medicine. 164(4):521-35.

19. EfthimiouJ BPJ. Effect of inhaled corticosteroids on bones and growth. European Respiratory Journal. 1998;11:1167-77.

20. Pruteanu Al, Chauhan BF, Zhang L, Prietsch SO, Ducharme FM. Inhaled corticosteroids in children with persistent asthma: effects on growth. Cochrane Database Systemic Review. 2014;7. https://doi.org/10.1002/ 14651858

21. Thomas BC, Stanhope R, Grant DB. Impaired growth in children with asthma during treatment with conventional doses of inhaled corticosteroids. Acta Paediatr. 1994;83(2):196-9.

22. Wright $\mathrm{CM}$, Cheetham $\mathrm{CD}$. The strengths and limitations of parental heights as a predictor of attained height. Arch Dis Child. 1999;81(3):257-60.

23. Axelsson I, Naumburg E, Prietsch SOM, Zhang L. Inhaled corticosteroids in children with persistent asthma: effects of different drugs and delivery devices on growth. Cochrane Database of Systematic Reviews 2019, Issue 6. Art. No.: CD010126.

24. Fatma D, Ozlem KARA, Derya TEPE, ESEN I. Bone Mineral density and vitamin $\mathrm{D}$ status in children and adolescents with congenital adrenal hyperplasi Turkish. J Med Sci. 2014:109-14.

25. Tse SM, Kelly HW, Litonjua AA, Van Natta ML, Weiss ST, Tantisira KG. Corticosteroid use and bone mineral accretion in children with asthma: effect modification by vitamin D. J Allergy Clinical Immunology. 2012:53-60.

26. Arend EE, Fischer GB, Debiasi M, Schmid H. Inhaled corticosteroid treatment and growth of asthmatic children seen at outpatient clinics. J Pediatr. 2006; 82(3):197-203.

27. Skoner DP. Inhaled corticosteroids Effects on growth and bone health. Alergy Asthma Immunology. 2016;117:595.

28. Allen HD, Thong IG, Clifton-Bligh P, Holmes S, Nery L, Wilson KB. Effects ofhigh-dose inhaled corticosteroids on bone metabolism in prepubertal childrenwith asthma. Pediatr Pulmonol. 2000;29:188e193.

29. Hopp RJ, Degan JA, Phelan J, Lappe J, Gallagher GC. Cross-sectional study of bone density in asthmatic children. Pediatr Pulmonol. 1995;20:189e192.

30. The Childhood Asthma Management Program Research Group. Longtermeffects of budesonide or nedocromil in children with asthma. N Engl J Med. 2000;343:1054-63.

31. Della Giustina A, Landi M, Bellini F, et al. Vitamin D, allergies and asthma: focus on pediatric patients. World Allergy Organ J. 2014;7(1):27.

\section{Publisher's Note}

Springer Nature remains neutral with regard to jurisdictional claims in published maps and institutional affiliations. 